\title{
Influence of Synthesis Route on the Radiation Sensing Properties of ZnO Nanostructures
}

\author{
R. A. Silva and M. O. Orlandi \\ Department of Physical Chemistry, São Paulo State University, 14800-060 Araraquara, SP, Brazil \\ Correspondence should be addressed to M. O. Orlandi; orlandi@iq.unesp.br
}

Received 20 August 2015; Revised 11 December 2015; Accepted 13 December 2015

Academic Editor: Ion Tiginyanu

Copyright (C) 2016 R. A. Silva and M. O. Orlandi. This is an open access article distributed under the Creative Commons Attribution License, which permits unrestricted use, distribution, and reproduction in any medium, provided the original work is properly cited.

\begin{abstract}
$\mathrm{ZnO}$ nanostructures were synthesized using two different routes and the light sensor response of structures was studied. The synthesis by carbothermal reduction resulted in $\mathrm{ZnO}$ tetrapods while the synthesis by microwave assisted hydrothermal method produced multipoint stars structures. Characterization by scanning and transmission electron microscopy confirmed that both structures consist of one-dimensional crystals with a hexagonal cross section and [001] growth direction. Under a simulated solar radiation spectrum, it was observed that tetrapods display a light sensor response of approximately 5000. For the multipoint stars, a maximum in the sensor signal value of 3400 was achieved, which also represents a substantial variation in the conductivity of the material. A model based on the surface oxygen presence is proposed to explain the observed results.
\end{abstract}

\section{Introduction}

The necessity for monitoring and the control of gas emission and ultraviolet (UV) radiation levels in the environment has been responsible for increasing the research and development of high performance sensor devices $[1,2]$. Sensors based on nanostructured semiconductor materials have been widely investigated due to their high response signal, high efficiency, and low processing cost [3-5]. Zinc oxide is a versatile material and when nanostructured, $\mathrm{ZnO}$ has potential applications in several areas, such as optoelectronics, varistors, sensors, transducers, and biomedical sciences [6-9]. $\mathrm{ZnO}$ is an n-type semiconductor with theoretical band gap of $3.3 \mathrm{eV}$ at room temperature and has several interesting properties: good transparency in the visible range of the electromagnetic spectrum, high electron mobility, piezoresponse, and luminescence, among others $[10,11]$. The high exciton binding energy $(60 \mathrm{meV})$ of the $\mathrm{ZnO}$ crystal can ensure efficient excitonic emission at room temperature; hence, luminescent properties are expected at room temperature with UV stimulation [12]. Moreover, the $\mathrm{ZnO}$ energy band gap corresponds to the energy of a photon in the ultraviolet range of spectrum, which indicates that this material is an excellent candidate for UV sensor devices [13].
UV sensors are used in many cases for the detection of ozone, monitoring UV solar radiation, fire alarms, and night vision devices [14]. Aiming to improve this class of sensors, studies have been conducted engineering structural and morphological changes to the $\mathrm{ZnO}$ materials [15-17].

Generally, the dimensions of nanostructures are comparable to the de Broglie wavelength, thereby revealing the quantum nature of the carriers present in the system. Due to confinement in these dimensions, carriers have a change in the occupation of energy states and may exhibit unusual electrical properties. In this way, the use of nanomaterials for sensor application can induce a fully depleted material resulting in a high sensor response once the dimensions are brought close to the Debye length of the material. Therefore, the synthesis route plays a crucial role in the final properties due to the morphological and chemical control of the material $[18,19]$.

In this work, $\mathrm{ZnO}$ nanostructures were prepared by both carbothermal reduction and microwave assisted hydrothermal methods and were characterized by XRD, UV-Vis, SEM, and TEM. The UV radiation sensor properties were tested in order to determine the effect of characteristic morphology of the material on its sensor response. 


\section{Experimental Methodology}

2.1. Preparation of $\mathrm{ZnO}$ Nanostructures. Two different methods were used to synthesize $\mathrm{ZnO}$ nanostructures: (i) an evaporation method using a carbothermal reduction process and (ii) a solution method using microwave assisted hydrothermal (MAH) synthesis [20-22].

For the evaporation synthesis method (i), a tubular furnace (EDG, model HT40) and high density alumina tubes were used. Commercial zinc oxide $(99.9 \%$ purity, SigmaAldrich) was first mechanically mixed with a carbon black reducing agent (Union Carbide, $>99 \%$ purity) in a 1:1 molar proportion. Next, one gram of the resulting mixture was placed in an alumina boat which was inserted in the central region (hot zone) of the tube furnace. A dynamic flow of inert gas $\left(\mathrm{N}_{2}\right)$ was kept constant during the synthesis to transport the vapor coming from the sublimation of the oxide to a cooler region of the tube, desublimating the vapor. The synthesis was carried out at $1100^{\circ} \mathrm{C}$ for 60 minutes with a heating rate of $10^{\circ} \mathrm{C} / \mathrm{min}$. A controlled amount of oxygen was introduced into the tube during the synthesis with a nitrogen: oxygen flux proportion of $80: 60$ to obtain the desired $\mathrm{ZnO}$ phase. The control of the morphology of the synthesized material was done by empirically controlling the supersaturation level of the vapor inside the tube, knowing that to obtain one-dimensional materials low levels of supersaturation are desirable [23].

In the MAH synthesis method (ii), an adapted microwave oven was used [24]. The precursors used for this synthesis were zinc acetate (99.99\% purity, Sigma-Aldrich) and sodium hydroxide ( $97.0 \%$ purity, Synth) in a mass ratio of $3: 10$. First, the zinc acetate was dissolved in deionized water by stirring and then sodium hydroxide was added in order to obtain a clear solution with a total volume of $130 \mathrm{~mL}$. Next, the solution was placed in a sealed Teflon reaction cell and the synthesis was carried out at $120^{\circ} \mathrm{C}$ for 20 minutes with a heating rate of $140^{\circ} \mathrm{C} / \mathrm{min}$. Then, the reactor was naturally cooled until reaching room temperature and the obtained particles were centrifuged several times with deionized water to achieve a neutral $\mathrm{pH}$.

2.2. Morphological and Structural Characterization. The morphological characteristics of the materials were studied by field emission scanning electron microscopy (FE-SEM, JEOL 7500F) and also by transmission electron microscopy (TEM, Philips, model CM200), both equipped with EDX. For the electron microscopy (EM) characterization, a sample suspension was prepared in isopropanol using an ultrasound bath to disperse the materials. Several drops of the suspension were deposited over a conductive Si substrate for SEM analyses, and one drop of the suspension was deposited on a carbon covered copper grid for TEM analysis. In both cases, the samples were dried at room temperature.

UV-Vis characterization was employed to estimate the band gap energy of the $\mathrm{ZnO}$ samples. Analyses were made using a Perkin Elmer spectrophotometer (LAMBDA 1050) in the wavelength range from 200 to $800 \mathrm{~nm}$, at room temperature.

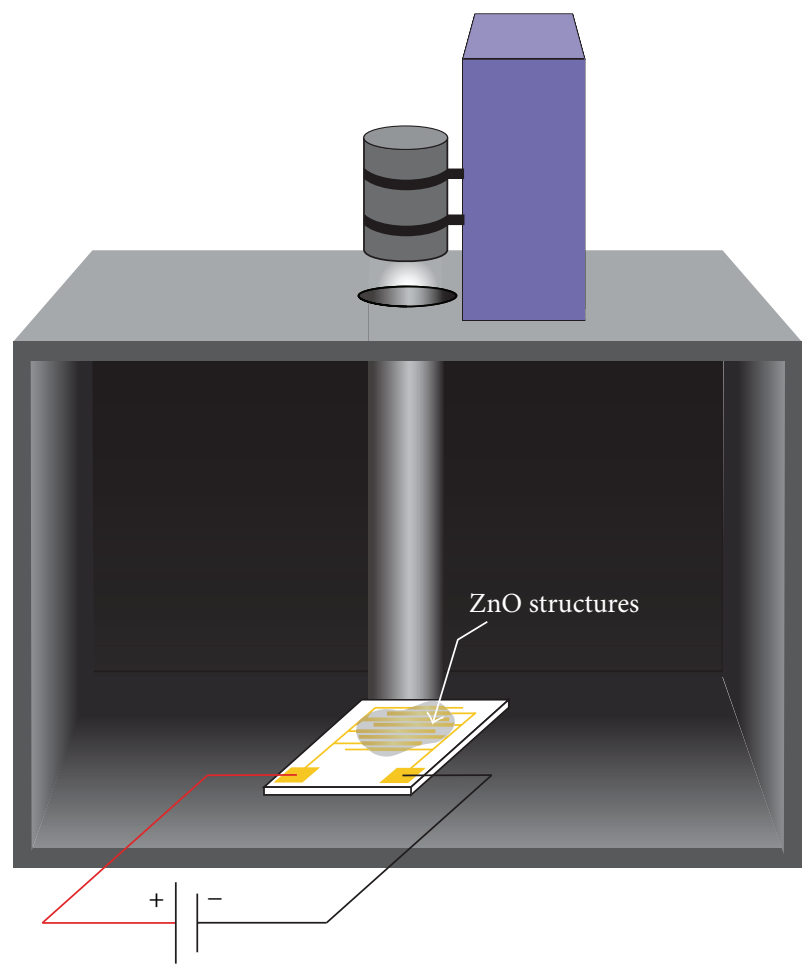

FIGURE 1: Obscure camera used to perform sensor measurements.

2.3. Light Sensor Studies. To perform the light sensor measurements, the synthesized materials were deposited on substrates with interdigitated gold electrodes. The substrate coated with nanostructures was inserted in an obscure camera with a shutter to allow the light to pass through (Figure 1). The substrate was positioned parallel to the shutter and perpendicular to the light beam from a xenon lamp (Solar Light, model 16S-300-VB; irradiance set at $50 \mathrm{~mW} / \mathrm{cm}^{2}$ ) which was positioned $40 \mathrm{~cm}$ from the sample. The materials were exposed to the radiation in cycles of 400 seconds, $200 \mathrm{~s}$ exposed followed by $200 \mathrm{~s}$ unexposed. The sensor measurements were carried out using a stabilized voltage source unit (Keithley, model 237). A constant $15 \mathrm{~V}$ bias was applied to the substrate while the current was monitored as a function of time. Based on the radiation sensor measurements, the sensor sensitivity, response time, recovery time, responsivity, and external quantum efficiency were calculated.

The sensitivity is quantified based on a relationship between the sensor response to a stimulus and the baseline, in this case the conductance when unexposed. Equation (1) was used to measure the sensor signal $(S)$ [25]:

$$
S=\frac{G_{L}}{G_{0}} \geq 1,
$$

where $G_{L}$ is the conductance with light incident on the material and $G_{0}$ is the conductance in the dark.

The parameters typically used to determine the speed of the sensor response are the response time and the recovery time. The response time is the time required to reach $90 \%$ of maximum sensor response after inserting the stimulus. The 
recovery time refers to the time the sensor needs to resume the sensor response to $90 \%$ of initial baseline (current with the sample in the dark) after the removal of stimulus.

Equation (2) is the mathematical expression for the response time $\left(t_{\text {resp. }}\right)$ [25]:

$$
t_{\text {resp. }}=\left(t_{\left[G_{0}+90 \%\left(G_{L}-G_{0}\right)\right]}\right)-t_{G_{0}} .
$$

\section{Results and Discussion}

3.1. Characterization of $\mathrm{ZnO}$ Nanostructures. XRD data from both samples (not shown here) showed that the structures were grown in the hexagonal $\mathrm{ZnO}$ structure (JCPDS \#361451) with no other peaks identified. Figure 2 shows SEM images of collected materials grown after the synthesis by the carbothermal reduction method. The material morphology is mostly composed of tetrapods, which have at their extremities long strands and a diameter less than $100 \mathrm{~nm}$. Some triangular plates can also be observed in the tangle of wires, and these plates are usually larger than $1 \mu \mathrm{m}$.

In order to study the tetrapods in more detail, transmission electron microscopy (TEM) was used and Figure 3(a) shows the TEM images of these $\mathrm{ZnO}$ structures. Based on the HRTEM analysis (Figure 3(b)), it is possible to observe the uniform growth of nanostructures without the occurrence of macroscopic defects along its length, meaning that the pods are monocrystalline. The interplanar distance of the crystalline planes in the growth direction has a value of $0.52 \mathrm{~nm}$, which is related to (001) plane of the $\mathrm{ZnO}$ structure (inset of Figure $3(\mathrm{~b})$ ). As these planes are rotated $90^{\circ}$ from the growth direction, it is possible to confirm that each pod grew in [001] direction, which is in agreement with previous studies of this material $[26,27]$. Moreover, it is known that $\mathrm{ZnO}$ materials grown in [001] direction have a hexagonal cross section, which agrees with the results shown in Figure 2.

Figure 4 shows SEM images of the structures obtained by MAH synthesis. The structures are composed of rods connected in a multipoint star morphology. Each rod has a hexagonal cross section, an average diameter of about $150 \mathrm{~nm}$, a smooth surface, and a faceted tip and is joined by a common point.

Figure 5 shows the base of a rod in a multipoint star structure. The insert shows a HRTEM image, where it is possible to observe a uniform growth without defects throughout the crystal. The observed interplanar distance has a value of $0.52 \mathrm{~nm}$ and is related to (001) plane of $\mathrm{ZnO}$. Similar to that observed for the tetrapod-like structures, the growth direction for each rod of the star morphology is [001].

The room temperature UV-Vis absorption spectra of the $\mathrm{ZnO}$ samples are shown in Figure 6, where it is possible to observe that the morphology difference plays no significant role in the absorption window. Absorption of both samples is very low from about $400 \mathrm{~nm}$ to $800 \mathrm{~nm}$, range which includes the visible spectrum. This result is expected since $\mathrm{ZnO}$ is known as a transparent semiconductor. Samples exhibit a strong absorption edge at about $370 \mathrm{~nm}$ which can be assigned to the intrinsic band gap absorption of $\mathrm{ZnO}$ due to electrons transition from the valence to the conduction band $\left(\mathrm{O}_{2 \mathrm{p}} \rightarrow \mathrm{Zn}_{3 \mathrm{~d}}\right)$ [28]. The absorption spectra show the
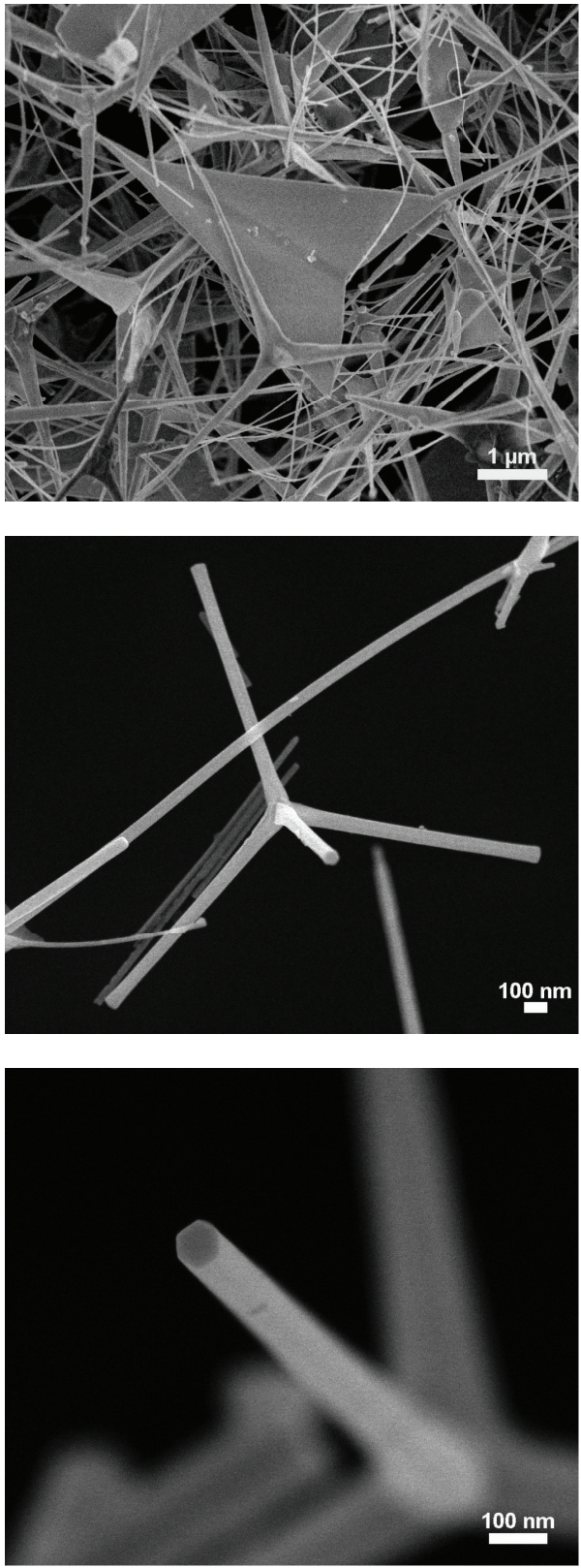

FIGURE 2: FEG-SEM images of $\mathrm{ZnO}$ tetrapods synthesized by the carbothermal reduction process.

cut-off wavelengths for the tetrapods and for the multipoint star samples at $368 \mathrm{~nm}$ and $370 \mathrm{~nm}$, respectively, blue shifted in relation to the bulk material, which is $380 \mathrm{~nm}$ [29]. This effect is attributed to the nanosize of the materials [30].

The optical energy gap can be determined using the Tauc plot (see (3)), which correlates the absorbance $(A)$ with the band gap energy $\left(E_{g}\right)[31]$ :

$$
(A h v)^{1 / n} \propto\left(h v-E_{g}\right) .
$$

In this equation, $h v$ is the photon energy and the value of the exponent $n$ denotes the nature of the sample transition. For direct allowed transitions, like in $\mathrm{ZnO}$ material, $n=1 / 2$. 


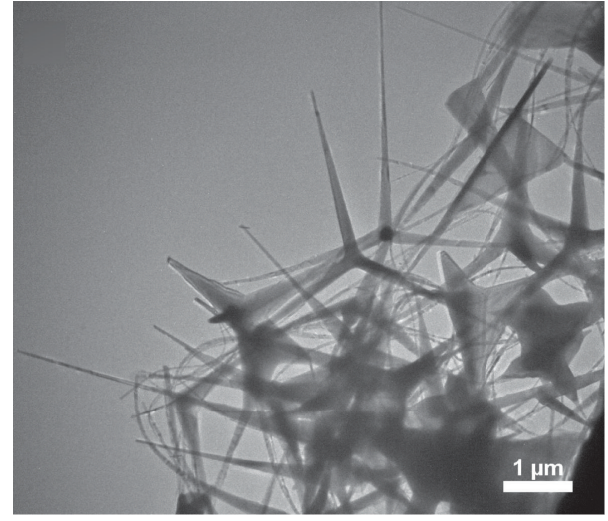

(a)

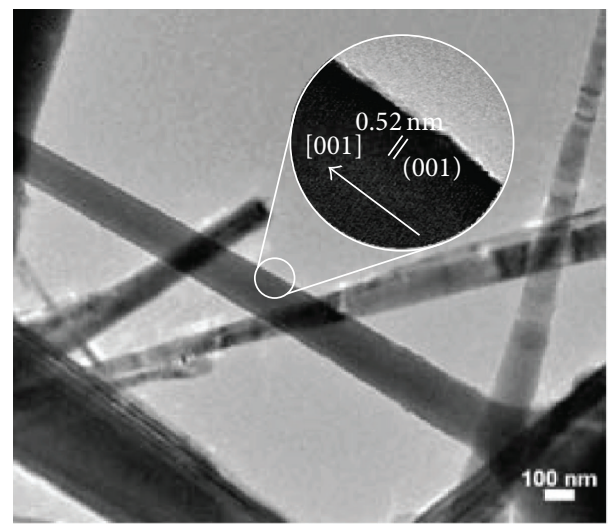

(b)

FIgURE 3: TEM images of (a) tetrapods structures and (b) image of an individual $\mathrm{ZnO}$ tetrapod. In the inset, a HRTEM image is shown.

From the inset of Figures 6(a)-6(b), it was possible to observe that the value of the optical band gap of both structures is about $\sim 3.2 \mathrm{eV}$, which is close to the expected band gap of the $\mathrm{ZnO}$ at room temperature.

3.2. Growth Mechanism of Structures. During the growth of the $\mathrm{ZnO}$ tetrapods by carbothermal evaporation method, no metal catalyst was needed and hence the growth is based on the vapor-solid (VS) mechanism. Because of the low supersaturation level associated with this process, it is common to obtain materials with a one-dimensional morphology. For the case of $\mathrm{ZnO}$, studies $[32,33]$ have shown that the low oxidation rate of $Z n$ vapor at the surface of $Z n$ particles allows the development of one-dimensional $\mathrm{ZnO}$ structures. The wurtzite crystal structure of hexagonal $\mathrm{ZnO}$ is composed of $\mathrm{O}^{2-}$ and $\mathrm{Zn}^{2+}$ ions arranged in a tetrahedral manner and stacked alternately along the $c$-axis direction. Wang et al. [34] showed that $\mathrm{ZnO}$ crystal growth is faster for (001) plane due to its self-catalytic property.

For the $\mathrm{ZnO}$ materials grown by $\mathrm{MAH}$, Huang et al. [35] proposed a suitable scheme for controlling the growth morphology. First, nuclei are generated by the induced microwave radiation (hot spots) and then progress to primary $\mathrm{ZnO}$ nanoparticles. Thus, the reaction continues to occur
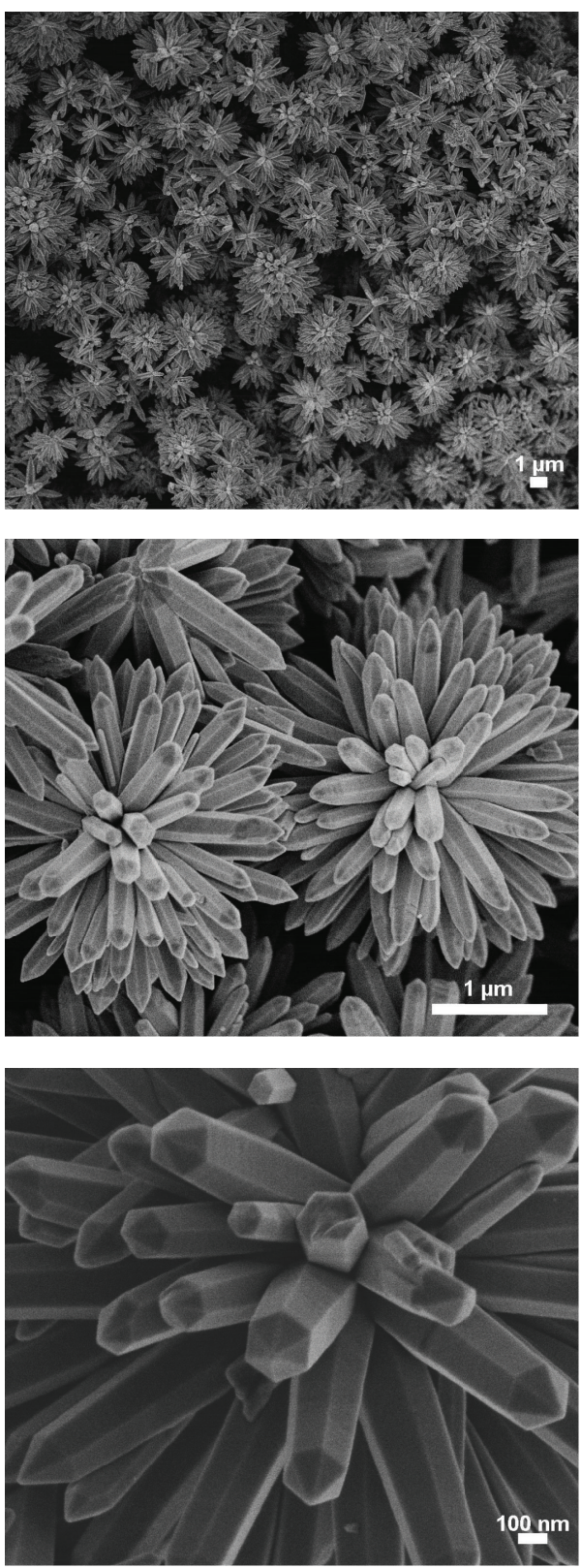

FIGURE 4: FEG-SEM images of $\mathrm{ZnO}$ stars obtained by the MAH method.

producing the growth of rods with hexagonal cross section. In order to form the star morphology, the nanorods need to grow from a common nucleus. Huang et al. suggest that this process may be related to an oriented attachment mechanism $[35,36]$ in which larger particles are grown from primary nanoparticles via an oriented binding process, wherein adjacent nanoparticles are self-organized due to a binding process which takes place when particles share a common crystallographic orientation. This model of growth can lead to the formation of faceted particles or anisotropic growth if there is sufficient difference in the surface energies of the different crystallographic faces. Therefore, it is believed that the formation of the $\mathrm{ZnO}$ complex nanostructures goes 


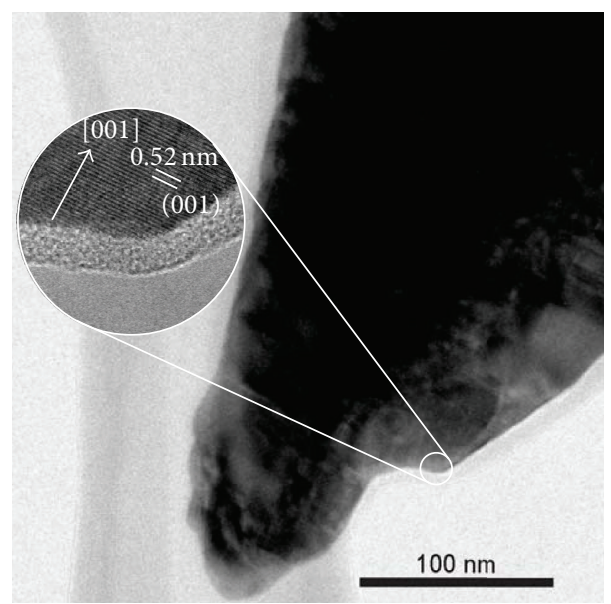

FIGURE 5: TEM image of an isolated rod of a multipoint star crystal. The insert picture shows the interplanar distance and the growth direction of the crystal.

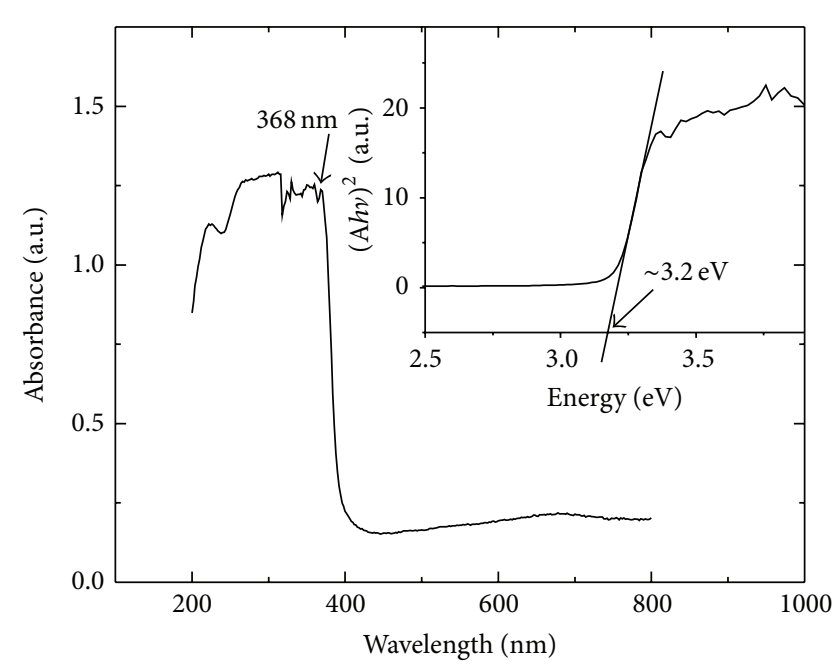

(a)

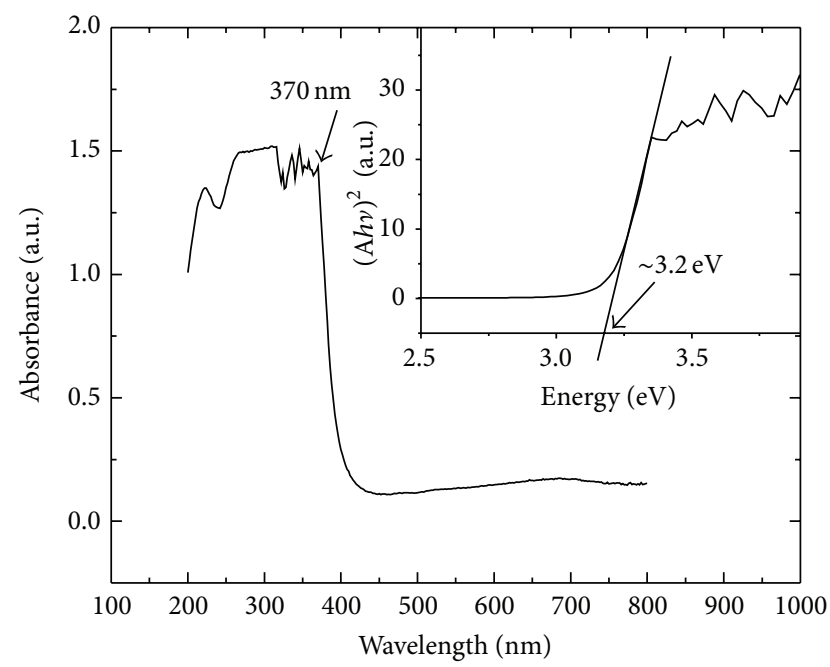

(b)

FIGURE 6: UV-Vis absorption spectra of ZnO nanoparticles: (a) tetrapod and (b) multipoint stars morphology. Inset shows the corresponding Tauc plot.

through three steps in sequence: (1) hydrolysis induced by a microwave, which leads to the formation of primary $\mathrm{ZnO}$ nanoparticles, (2) fusion of primary nanoparticles following oriented growth to form nanorods structures, and (3) a process of growth and crystallization, giving rise to the formation of the final morphology (multipoint stars).

In order to achieve such a level of surface uniformity, it is possible that dissociation/recrystallization processes occur during the synthesis.

3.3. Sensor Properties. The operating principle of the radiation sensor is based on the adsorption of oxygen on the surface of the material. In the absence of light, oxygen molecules adsorb on the surface of the nanostructures by chemisorbing free electrons from the semiconductor material, turning into negatively charged ions. The adsorption of oxygen is generally more effective for relatively high working temperatures, from 100 to $500^{\circ} \mathrm{C}$, where the interaction of oxygen through the ionosorption mechanism may occur as molecular species $\left(\mathrm{O}_{2}^{-}\right)$and/or atomic $\left(\mathrm{O}^{-}, \mathrm{O}^{2-}\right)$ species. Using different techniques, such as Temperature Programmed Desorption (TPD), Fourier Transform Infrared (FTIR) spectroscopy, and Electron Spin Resonance (ESR), it was shown that below $150^{\circ} \mathrm{C}$ the predominant species are the molecular ones while above this temperature the atomic species dominate [37]. The chemisorption of free electrons on the semiconductor surface enlarges the depletion layer (Figure 7) resulting in a decrease of the semiconductor conductivity. The following chemical reaction explains the effect of decreasing the number of free charge carriers on the material by the chemisorption process:

$$
\mathrm{O}_{2(\mathrm{~g})}+\mathrm{e}^{-} \longrightarrow \mathrm{O}_{2}^{-}(\mathrm{ad})
$$



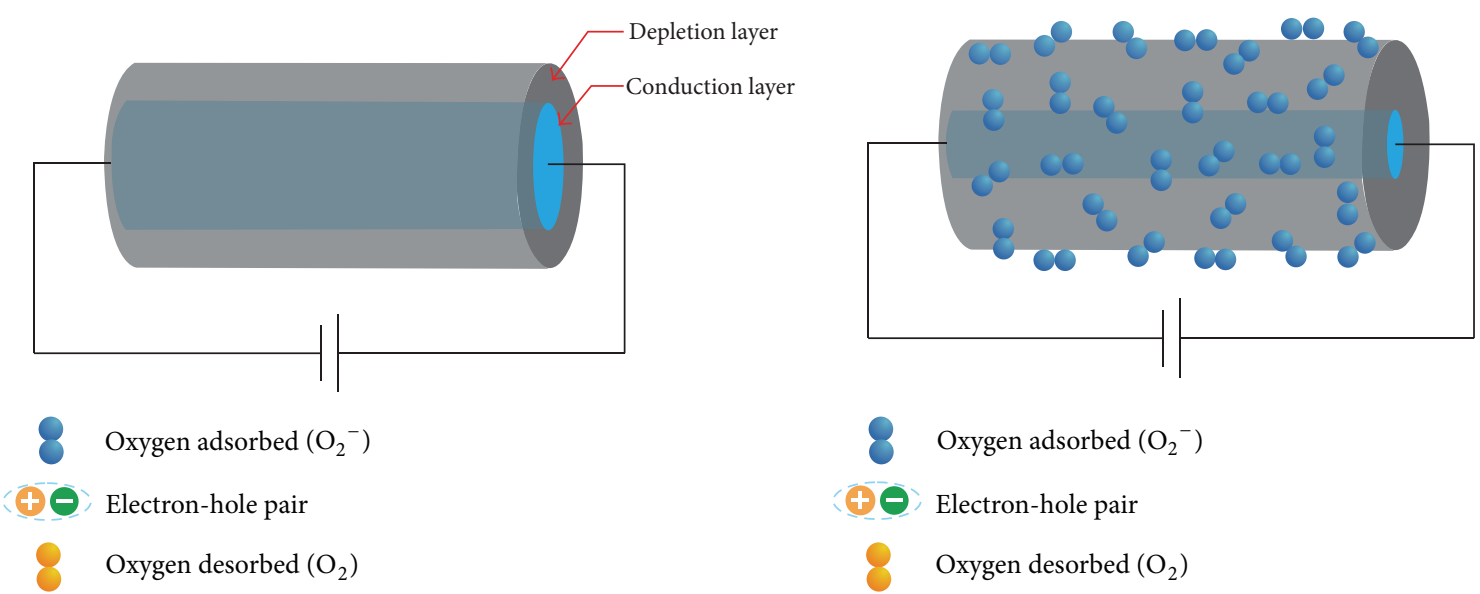

(a)

(b)

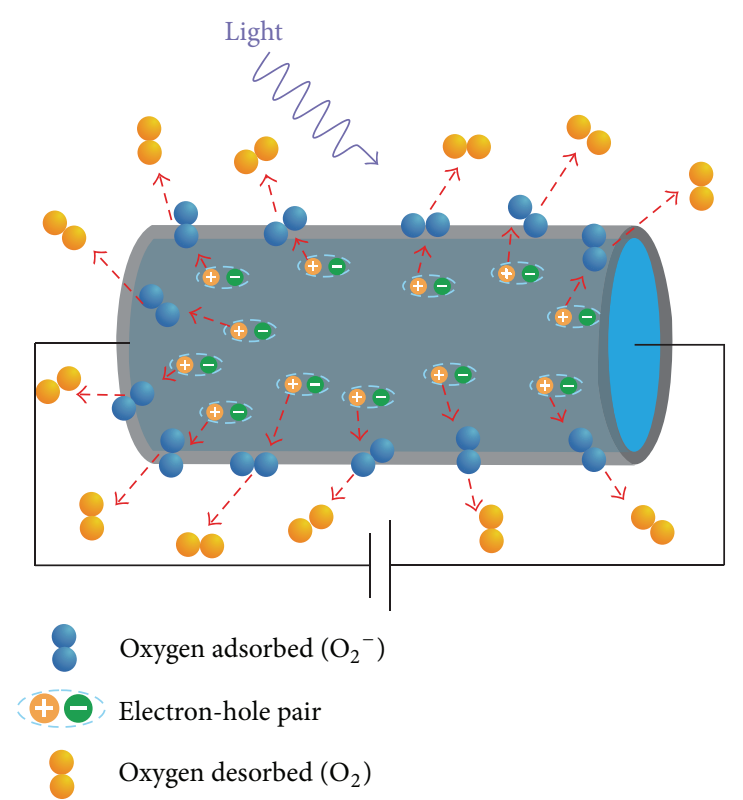

(c)

FIGURE 7: Schematic diagram of the depletion layer in $\mathrm{ZnO}$ : (a) without the oxygen adsorption, (b) with adsorption of oxygen from the ambient atmosphere, and (c) with incidence of light.

After exposure to UV light, electrons and holes are photogenerated in the material surface because the $\mathrm{ZnO}$ band gap is in the UV light energy range. Then, these holes at the surface of the material discharge the adsorbed oxygen ions through the following recombination:

$$
\mathrm{h}^{+}+\mathrm{O}_{2}^{-}(\mathrm{ad}) \longrightarrow \mathrm{O}_{2(\mathrm{~g})}
$$

Due to the reaction of holes with the surface ions, the photogenerated electrons will not completely recombine, and some of them become free electrons. This phenomenon increases the conductance of the semiconductor nanostructure when exposed to the light, as observed in our results. This suggests that photoelectric gain propagation is operating in the semiconductor rather than a simple process of capturing the light. When the light is off, the photogenerated electrons can still contribute to the current until they are captured by the surface states (e.g., through an oxygen chemisorption process). Then, it is expected that nanostructures can further increase the sensitivity of the material due to an increased surface area, which can ideally lead to the recognition of single photon detection [38, 39]. Moreover, the photoresponse is strongly dependent on the environment gas and is very slow in vacuum and in inert gases, presenting the response known as persistent photoconductivity, and fast in air [40]. As stated before, it should be related to the oxygen presence on the material surface.

Figure 8(a) presents the electrical response of $\mathrm{ZnO}$ structures obtained by the carbothermal reduction and by $\mathrm{MAH}$ processes during the light sensor cycles. It can be observed that when the light is turned on the sample conductance increases sharply and then gradually becomes saturated. After the removal of the light stimulus, the conductance tends to 


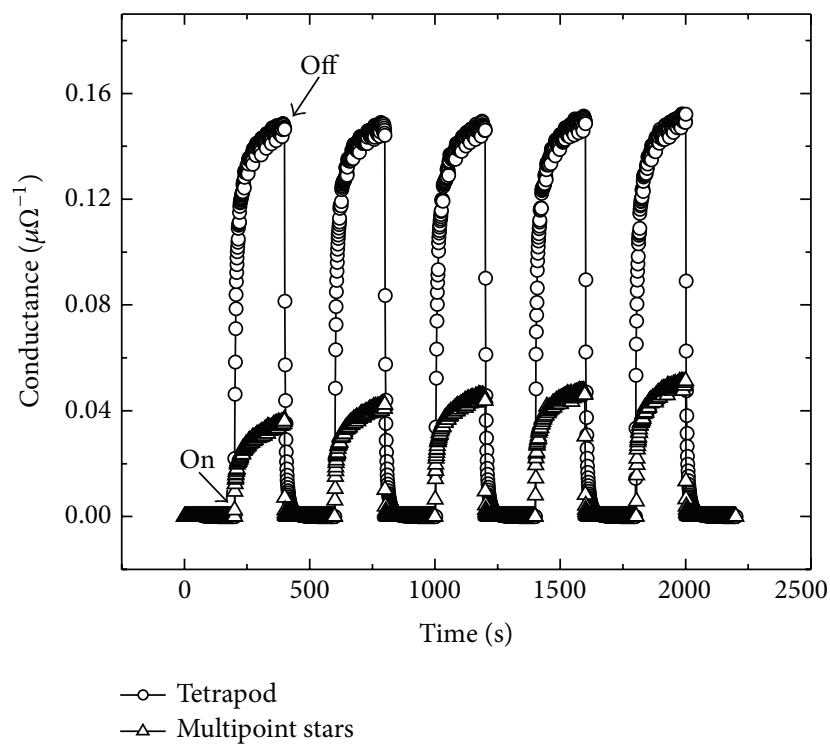

(a)

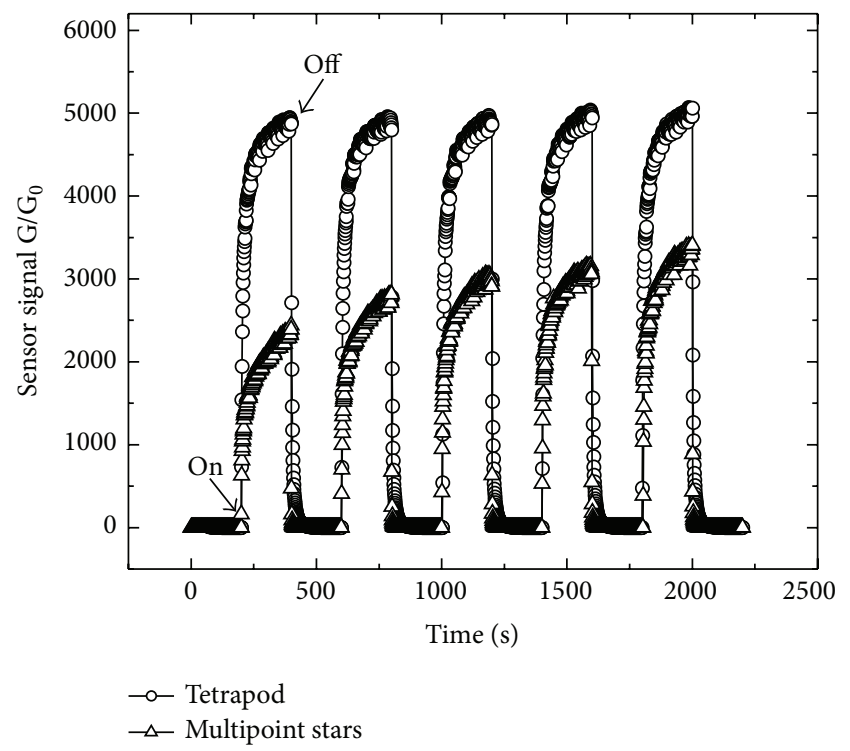

(b)

Figure 8: (a) Photoconductive response of the tetrapods and multipoint stars structures. (b) Light sensor signal of $\mathrm{ZnO}$ tetrapods and multipoint stars.

return to its initial state, in an exponential fashion. Several exposition cycles were performed and it can be observed for the tetrapods that the curves have good repeatability over time, reaching the same level of conductance when light is triggered on and maintained for 200 seconds. This result shows that the $\mathrm{ZnO}$ tetrapods are stable even after several cycles of light exposition, which is an important result for practical applications.

The conductance versus time for the $\mathrm{ZnO}$ multipoint stars obtained by the MAH synthesis (Figure 8(a)) also presents a good response to the light stimulus, but in these curves the conductance is observed to increase as a function of time. Despite the occurrence of this shift, the value of the response of the material is also significant. Furthermore, it was observed that after removal of the stimulus, the conductance always returned to the same value. In other words, the offset drift recovery is very low.

The sensor response of the tetrapods and the multipoint stars samples is presented in Figure 8(b). For the tetrapods, the sensor signal was calculated to be 5000 , which is a very high value of light sensor signal. The average response time of this sensor was 43 seconds, which is reasonable regarding the level of the sensor response achieved.

For the multipoint stars sample, the sensor signal was approximately 2500 for the lower curve and 3400 for the bigger one, with respective response times of 120 and $60 \mathrm{sec}-$ onds, showing that it is not very stable over time. Again, the obtained sensor signal values are very high when compared to those found in the literature for other $\mathrm{ZnO}$ structures [4144].

Table 1 presents the values of the sensor signal, response time, recovery time, responsivity, and external quantum efficiency of the tetrapod and multipoint stars $\mathrm{ZnO}$ samples.
TABLE 1: Sensor signal, response time, and recovery time of the $\mathrm{ZnO}$ tetrapods and multipoint stars devices.

\begin{tabular}{lcc}
\hline & ZnO tetrapod & ZnO multipoint stars \\
\hline Sensor signal & 5000 & $2400-3400$ \\
Response time (s) & 43 & $120-60$ \\
Recovery time (s) & 10 & $4-2$ \\
\hline
\end{tabular}

Possibly, the instability of the multipoint star sample can be related to the fact that its morphology is "closed," thereby preventing the entry of light and thus reducing the formation of electron-hole pairs in the material with time. This also affects the heating of the entire material over time, which hinders the desorption of oxygen molecules, since studies indicate that the main mechanism of desorption of a material under light incidence is the local heating [45].

So, in this work, we have showed the synthesis and light sensor characterization of $\mathrm{ZnO}$ nanostructures produced by carbothermal reduction process and microwave assisted hydrothermal method. Results show that both systems have a very high sensor signal when exposed to light, but tetrapods grown by the carbothermal method are the most sensitive and more stable over time. Models of the materials growth and light sensor response were proposed and additional studies with decorated structures may be done in the future to further increase the sensor response of materials.

\section{Conclusion}

In this work, two distinct synthesis methods were used to produce $\mathrm{ZnO}$ crystals and the light sensor response of materials was studied. Using the carbothermal reduction method, 
a tetrapod morphology was obtained and the microwave assisted hydrothermal method generated a multipoint starlike morphology. The synthesis methods were effective in obtaining pure $\mathrm{ZnO}$ without impurities and with a uniform morphology, in both methods. Both samples showed good response to solar radiation, and the sensor signal obtained was 5000 for the tetrapods. For the multipoint stars, there was a gradual increase of the signal and its value ranges from approximately 2400 to 3400 . The instability of the multipoint star sample was attributed to its closed morphology. Models based on oxygen adsorption on the material surface were addressed to explain the sensor response of materials.

\section{Conflict of Interests}

The authors declare that there is no conflict of interests regarding the publication of this paper.

\section{Acknowledgments}

The authors would like to thank the funding agencies FAPESP (Proc. 2013/07296-2) and CNPq (Proc. 447760/2014$9,309013 / 2012-7$, and 555475/2009-3) for the financial support. SEM and TEM facilities were provided by the LMA-IQUNESP. They are also thankful to George Harrington for his contribution to this work.

\section{References}

[1] H. Kind, H. Yan, B. Messer, M. Law, and P. Yang, "Nanowire ultraviolet photodetectors and optical switches," Advanced Materials, vol. 14, no. 2, pp. 158-160, 2002.

[2] O. Lupan, L. Chow, G. Chai, L. Chernyak, O. LopatiukTirpak, and H. Heinrich, "Focused-ion-beam fabrication of $\mathrm{ZnO}$ nanorod-based UV photodetector using the in-situ lift-out technique," Physica Status Solidi (A) Applications and Materials Science, vol. 205, no. 11, pp. 2673-2678, 2008.

[3] J.-M. Jang, C.-R. Kim, H. Ryu, M. Razeghi, and W.-G. Jung, "ZnO 3D flower-like nanostructure synthesized on GaN epitaxial layer by simple route hydrothermal process," Journal of Alloys and Compounds, vol. 463, no. 1-2, pp. 503-510, 2008.

[4] L. Sang, M. Liao, and M. Sumiya, "A comprehensive review of semiconductor ultraviolet photodetectors: from thin film to one-dimensional nanostructures," Sensors, vol. 13, no. 8, pp. 10482-10518, 2013.

[5] S. W. Koch, T. Meier, W. Hoyer, and M. Kira, "Theory of the optical properties of semiconductor nanostructures," Physica E: Low-Dimensional Systems and Nanostructures, vol. 14, no. 1-2, pp. 45-52, 2002.

[6] A. B. Djuriić, A. M. C. Ng, and X. Y. Chen, "ZnO nanostructures for optoelectronics: material properties and device applications," Progress in Quantum Electronics, vol. 34, no. 4, pp. 191-259, 2010.

[7] J. M. Cannata, J. A. Williams, Q. F. Zhou et al., "Self-focused $\mathrm{ZnO}$ transducers for ultrasonic biomicroscopy," Journal of Applied Physics, vol. 103, no. 8, Article ID 084109, 2008.

[8] Y. Xie, Y. Wang, T. Zhang, G. Ren, and Z. Yang, "Effects of nanoparticle zinc oxide on spatial cognition and synaptic plasticity in mice with depressive-like behaviors," Journal of Biomedical Science, vol. 19, no. 1, article 14, 2012.
[9] S. K. Gupta, A. Joshi, and K. Manmeet, "Development of gas sensors using $\mathrm{ZnO}$ nanostructures," Journal of Chemical Sciences, vol. 122, no. 1, pp. 57-62, 2010.

[10] Ü. Özgür, Y. I. Alivov, C. Liu et al., "A comprehensive review of $\mathrm{ZnO}$ materials and devices," Journal of Applied Physics, vol. 98, no. 4, Article ID 041301, 2005.

[11] M. Sui, P. Gong, and X. Gu, "Review on one-dimensional $\mathrm{ZnO}$ nanostructures for electron field emitters," Frontiers of Optoelectronics, vol. 6, no. 4, pp. 386-412, 2013.

[12] Z. L. Wang, "Zinc oxide nanostructures: growth, properties and applications," Journal of Physics Condensed Matter, vol. 16, no. 25, pp. R829-R858, 2004.

[13] S. Liang, H. Sheng, Y. Liu, Z. Huo, Y. Lu, and H. Shen, "ZnO Schottky ultraviolet photodetectors," Journal of Crystal Growth, vol. 225, no. 2-4, pp. 110-113, 2001.

[14] M. Razeghi and A. Rogalski, "Semiconductor ultraviolet detectors," Journal of Applied Physics, vol. 79, no. 10, pp. 7433-7473, 1996.

[15] J. Cheng, Y. Zhang, and R. Guo, "ZnO microtube ultraviolet detectors," Journal of Crystal Growth, vol. 310, no. 1, pp. 57-61, 2008.

[16] H. K. Yadav, K. Sreenivas, and V. Gupta, "Enhanced response from metal/ZnO bilayer ultraviolet photodetector," Applied Physics Letters, vol. 90, no. 17, Article ID 172113, 2007.

[17] H. K. Yadav, K. Sreenivas, and V. Gupta, "Study of metal/ZnO based thin film ultraviolet photodetectors: the effect of induced charges on the dynamics of photoconductivity relaxation," Journal of Applied Physics, vol. 107, no. 4, Article ID 044507, 2010.

[18] J. Hu, T. W. Odom, and C. M. Lieber, "Chemistry and physics in one dimension: synthesis and properties of nanowires and nanotubes," Accounts of Chemical Research, vol. 32, no. 5, pp. 435-445, 1999.

[19] B. D. Yao, Y. F. Chan, and N. Wang, "Formation of ZnO nanostructures by a simple way of thermal evaporation," Applied Physics Letters, vol. 81, no. 4, pp. 757-759, 2002.

[20] Y. S. Lim, J. W. Park, M. S. Kim, and J. Kim, "Effect of carbon source on the carbothermal reduction for the fabrication of ZnO nanostructure," Applied Surface Science, vol. 253, no. 3, pp. 1601-1605, 2006.

[21] B. V. L'vov, "Mechanism of carbothermal reduction of iron, cobalt, nickel and copper oxides," Thermochimica Acta, vol. 360, no. 2, pp. 109-120, 2000.

[22] S.-S. Wu, Q.-M. Jia, Y.-L. Sun, S.-Y. Shan, L.-H. Jiang, and Y.M. Wang, "Microwave-hydrothermal preparation of flower-like $\mathrm{ZnO}$ microstructure and its photocatalytic activity," Transactions of Nonferrous Metals Society of China (English Edition), vol. 22, no. 10, pp. 2465-2470, 2012.

[23] A. P. Levitt, Whisker Technology, Wiley-Interscience, 1970.

[24] M. L. Moreira, G. P. Mambrini, D. P. Volanti et al., "Hydrothermal microwave: a new route to obtain photoluminescent crystalline $\mathrm{BaTiO}_{3}$ nanoparticles," Chemistry of Materials, vol. 20, no. 16, pp. 5381-5387, 2008.

[25] J. L. G. Fierro, Metal Oxides: Chemistry and Applications, CRC Press, 2005.

[26] J. F. Gong, H. B. Huang, Z. Q. Wang, X. N. Zhao, S. G. Yang, and Z.-Z. Yu, "A third kind growth model of tetrapod: rodbased single crystal $\mathrm{ZnO}$ tetrapod nanostructure," Materials Chemistry and Physics, vol. 112, no. 3, pp. 749-752, 2008.

[27] L. Feng, A. Liu, M. Liu, Y. Ma, J. Wei, and B. Man, "Fabrication and characterization of tetrapod-like $\mathrm{ZnO}$ nanostructures prepared by catalyst-free thermal evaporation," Materials Characterization, vol. 61, no. 1, pp. 128-133, 2010. 
[28] A. K. Zak, M. E. Abrishami, W. H. A. Majid, R. Yousefi, and S. M. Hosseini, "Effects of annealing temperature on some structural and optical properties of $\mathrm{ZnO}$ nanoparticles prepared by a modified sol-gel combustion method," Ceramics International, vol. 37, no. 1, pp. 393-398, 2011.

[29] E. G. Goh, X. Xu, and P. G. McCormick, "Effect of particle size on the UV absorbance of zinc oxide nanoparticles," Scripta Materialia, vol. 78-79, pp. 49-52, 2014.

[30] S. S. Kumar, P. Venkateswarlu, V. R. Rao, and G. N. Rao, "Synthesis, characterization and optical properties of zinc oxide nanoparticles," International Nano Letters, vol. 3, no. 1, article 30, 2013.

[31] J. Tauc, "Absorption edge and internal electric fields in amorphous semiconductors," Materials Research Bulletin, vol. 5, no. 8, pp. 721-729, 1970.

[32] N.-K. Park, G. B. Han, J. D. Lee et al., “The growth of ZnO nanowire by a thermal evaporation method with very small amount of oxygen," Current Applied Physics, vol. 6, supplement 1, pp. el76-e181, 2006.

[33] D. H. Liu, L. Liao, J. C. Li, H. X. Guo, and Q. Fu, "Preparation and photoluminescence of $\mathrm{ZnO}$ nanostructures by thermal evaporation growth without catalysts," Materials Science and Engineering B, vol. 121, no. 1-2, pp. 77-80, 2005.

[34] Z. L. Wang, X. Y. Kong, and J. M. Zuo, "Induced growth of asymmetric nanocantilever arrays on polar surfaces," Physical Review Letters, vol. 91, no. 18, Article ID 185502, 2003.

[35] J. Huang, C. Xia, L. Cao, and X. Zeng, "Facile microwave hydrothermal synthesis of zinc oxide one-dimensional nanostructure with three-dimensional morphology," Materials Science and Engineering B: Solid-State Materials for Advanced Technology, vol. 150, no. 3, pp. 187-193, 2008.

[36] R. L. Penn and J. F. Banfield, "Imperfect oriented attachment: dislocation generation in defect-free nanocrystals," Science, vol. 281, no. 5379, pp. 969-971, 1998.

[37] A. Gurlo, U. Wiemar, and N. Barsan, "Gas sensors based on semiconducting metal oxides," in Metal Oxides: Chemistry and Applications, Chemical Industries, pp. 683-738, CRC Press, New York, NY, USA, 2005.

[38] X. B. Wang, Y. Q. Liu, and D. B. Zhu, "Two- and threedimensional alignment and patterning of carbon nanotubes," Advanced Materials, vol. 14, no. 2, pp. 165-167, 2002.

[39] J. H. Jun, H. Seong, K. Cho, B.-M. Moon, and S. Kim, "Ultraviolet photodetectors based on $\mathrm{ZnO}$ nanoparticles," Ceramics International, vol. 35, no. 7, pp. 2797-2801, 2009.

[40] M. Liu and H. K. Kim, "Ultraviolet detection with ultrathin $\mathrm{ZnO}$ epitaxial films treated with oxygen plasma," Applied Physics Letters, vol. 84, no. 2, pp. 173-175, 2004.

[41] N. K. Hassan and M. R. Hashim, "Flake-like ZnO nanostructures density for improved absorption using electrochemical deposition in UV detection," Journal of Alloys and Compounds, vol. 577, pp. 491-497, 2013.

[42] Z. Bai, X. Yan, X. Chen, K. Zhao, P. Lin, and Y. Zhang, "High sensitivity, fast speed and self-powered ultraviolet photodetectors based on $\mathrm{ZnO}$ micro/nanowire networks," Progress in Natural Science: Materials International, vol. 24, no. 1, pp. 1-5, 2014.

[43] G.-H. Shen and F. C.-N. Hong, "Ultraviolet photosensors fabricated with $\mathrm{Ag}$ nanowires coated with $\mathrm{ZnO}$ nanoparticles," Thin Solid Films, vol. 570, part B, pp. 363-370, 2014.

[44] Y. Sun, X. Zhang, C. Tian, D. Jiang, Z. Li, and S. Liu, "Varying load resistances of zinc oxide ultraviolet photodetectors: a simple method to change responsivity," Materials Science in Semiconductor Processing, vol. 31, pp. 10-13, 2015.
[45] G. A. Somorjai, "Surface reconstruction and catalysis," Annual Review of Physical Chemistry, vol. 45, no. 1, pp. 721-751, 1994. 

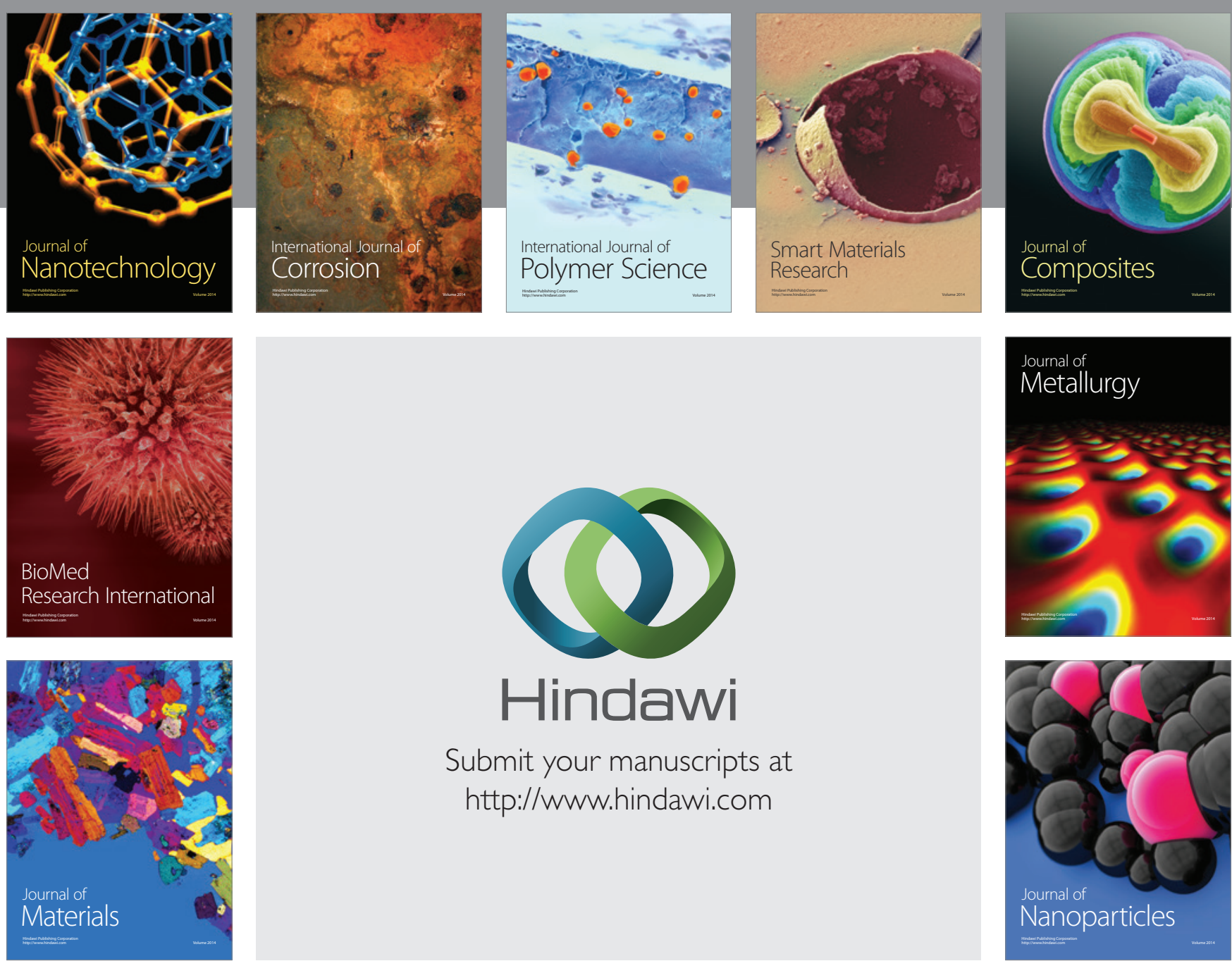

\section{Hindawi}

Submit your manuscripts at

http://www.hindawi.com

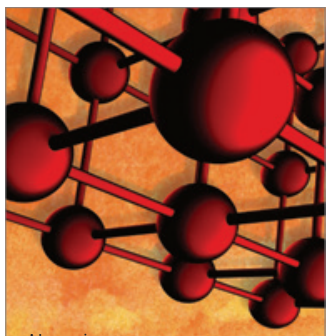

Materials Science and Engineering
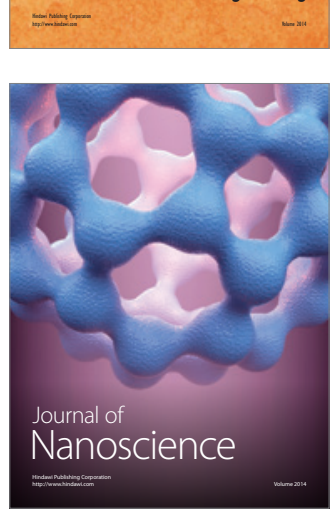
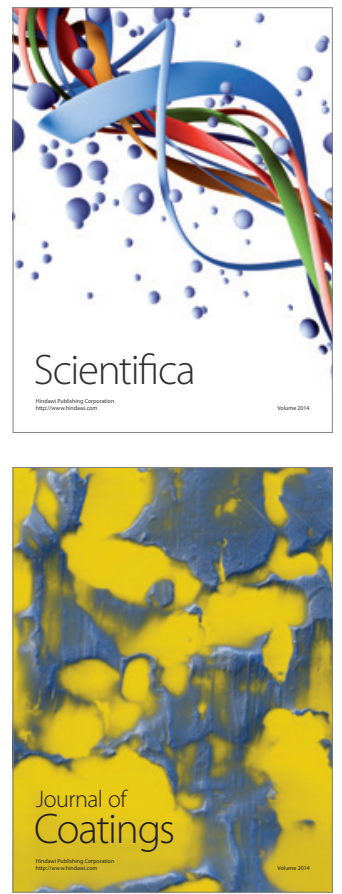
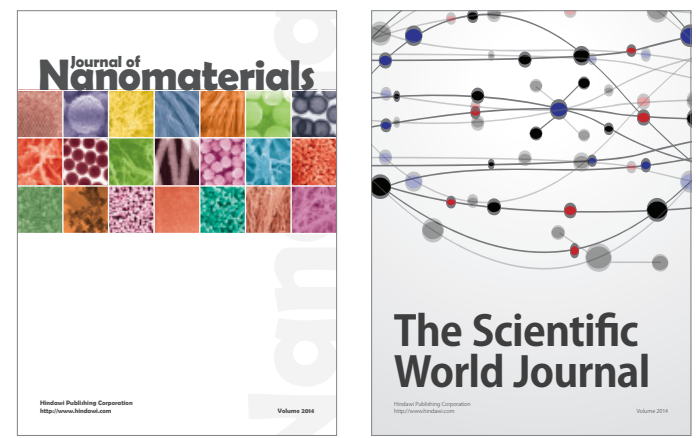

The Scientific World Journal
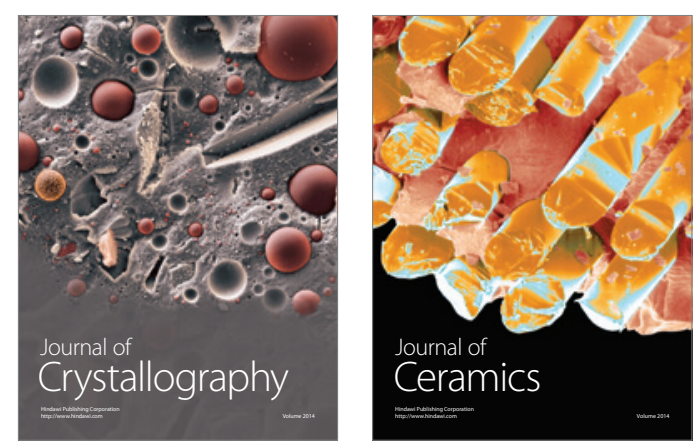
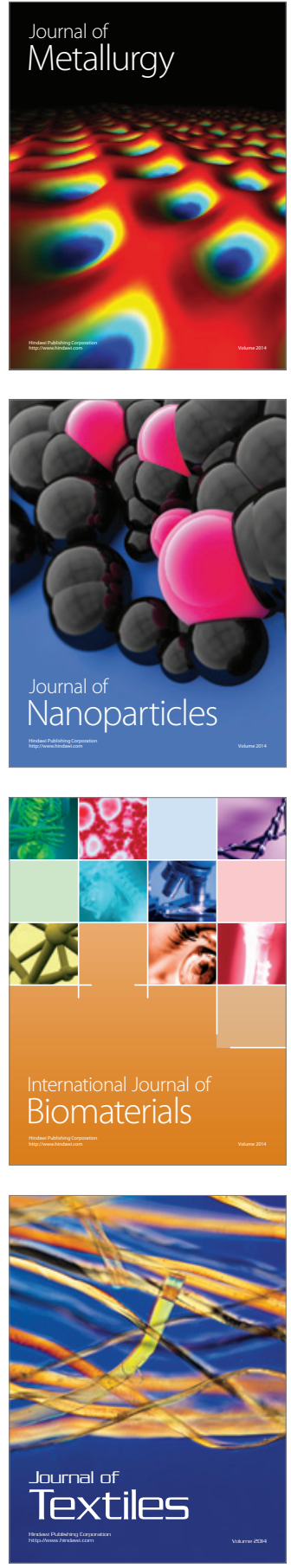